\title{
Implementation of Cloud Technology for Remote Data Access for Library Management using ARM11
}

\author{
Sameer R. Karadkar \\ M. Tech student \\ Department of Technology \\ Shivaji University \\ Kolhapur, Maharashtra, India
}

\author{
Uttam L. Bombale, Ph.D \\ Associate professor \\ Department of Technology \\ Shivaji University \\ Kolhapur, Maharashtra, India
}

\begin{abstract}
Cloud storage has been a recent topic of technology in which the most applications are being taken place because of its inherent advantages. The cloud storage could be used to optimize the places like Libraries on which this paper mainly focuses on. Library has always been an area of optimization in terms of the flaws and drawbacks of conventional systems. With the revolutions in the field of technology, Library also has undergone various improvements in terms of management. The time always demands the use of best available technology for higher efficiencies. Keeping that perspective, a modern Library management system is introduced, that overcomes all the previous system flaws using latest technology available. The proposed system makes use of modern concepts and technologies like RFID identification, Wiegand protocol, ARM 11 controller, Cloud etc.to make the management task easier and efficient compared to systems being implemented today. The system is designed to reduce the unnecessary manual work being carried out at libraries, to remove the costlier counterparts being employed which are not only adding to costs but also in terms of space required to perform their tasks, to have a simple and transparent access to the library data to both authorities and beneficiaries'.
\end{abstract}

\section{General Terms}

RFID identification, Wiegand protocol, ARM 11 controller, Cloud, Raspberry PI .

\section{Keywords}

Cloud, storage, Library management, RFID technology, Raspberry PI

\section{INTRODUCTION}

Cloud computing is a combination of many computing fields and has gained much popularity in the recent years. Cloud computing has in it, computing, storage, services, and applications over the Internet. Moreover, cloud computing helps to reduce capital cost and provides flexibility in terms of resource allocations where required [1]. Cloud is a technology of which the basic roots can be found in distributed computing, grid computing, service orientation, virtualization and market oriented computing. Cloud services facilitate us in various ways which include infrastructure such as storage servers, platforms such as operating systems, and application software [2]. The proposed system makes use of this technology for optimizing libraries data management to achieve a modern technological touch and in turn to achieve an efficient system. Whereas another technology called Radio frequency identification (RFID) is playing a vital role in modern identification techniques. RFID technology i.e. Radio Frequency Identification technology makes use of radio frequencies to identify particular objects and distinguish them from others. RFID being reliable and efficient, it is making its way into various identification tasks to reduce the drawbacks of its conventional counterparts [3]. This RFID technology is used in the proposed system to make the identification more reliable and robust. And the third part of the system is ARM11 controller which is the processing part. The proposed system makes use of cloud concept for remote data access and management. It makes use of this cloud and RFID for more secure and efficient library data management. The system keep records of books, Borrowers name, ID etc. using RFID identification technology and consequently provide those data onto cloud. The authenticated user can then access the information which is being sent on the cloud and so as the student using its own given authenticated data like User ID and password. The proposed system is divided mainly into three parts logically

1. RFID reader and its corresponding controller circuitry

2. ARM 11

3. Cloud

The first part is RFID part. In this part, system makes use of RFID identification property. It uses RFID tags and RFID reader for the task. Each RFID tag will be having its own unique ID which will be decoded in terms of its corresponding book's specific information such as name, author, publication etc. These tags are attached to corresponding books. There would be other tags too being provided to users of library. Similar to book RFID tags, these tags will be decoded in terms of the identification of particular user, using unique identification scheme of RFID technology. There would be one RFID reader which will be used to read these RFID tags. The RFID reader will read the corresponding tags of the book and the user and hence it will provide this information to its corresponding controller unit. The controller unit will get this information and will show it on the LCD whether the tag is successfully detected or not and will decide on whether to in this book or to out using keypad connected to it. The ARM11 will take care of further processing of data sent by controller. The ARM will take care of processing in the system. It will be having USB ports as well as SD card facility. The ARM will be having Ethernet port connected to it. The ARM will be configured to send data on cloud using Ethernet port. The third part is cloud. The data manipulated by ARM11 for transmission will be sent on cloud. The data will remain on cloud until anyone tries to access it. System will be deployed here to facilitate authenticated person like librarian to access all details including which books are issued to which borrowers and available books in library. Apart from these, authenticated students, professors of that institute can access 
cropped data like name list of currently available books, in the library. The benefit would be to librarian to view the current status of library from anywhere like home etc. having no restriction of place or to students and professors to know about the currently available literature anytime anywhere so it will reduce the time wastage which occurs when students or professors approach the library and they find the unavailability of required books. Further database management is done using RFID technology \& CLOUD technology which are having inherent advantages over conventional systems as explained above. The biggest advantage being the replacement of big computers used to keep record of data from libraries by ARM11.

\section{BACKGROUND}

Mobile cloud application issues are addressed with elaboration of mobile cloud application models to implement this highly advanced and beneficial technology to be supported by mobile devices [1]. Introduction of cloud and best available literature has been described along with different introductory aspects of cloud [2]. Study of survivability issues related to RFID systems and survey of existing techniques whose usability and adaptability toward survivability would be beneficial is addressed [3]. Limitations and opportunities of deploying data management issues on the cloud computing platform is addressed [4]. Cloud is studied in terms of optimization of existing tedious systems to yield efficiency of work in places like Government organizations for cost reduction and efficiency [5]. Study regarding the mechanism of sensing in RFID tags and corresponding issues in the RFID technology are addressed [6]. The measure for accuracy and precision in a RFID localization system with regard to RFID tag distribution in RFID technology is addressed [7]. A conventional system using RFID technology for library management is referred which uses IoT i.e. internet of things and Motorola MC 9090 scanner is used for the task [8]. Earth observation data processing using Web Processing Service is implemented using Cloud technology for its inherent advantages. It is a specific application of cloud computing being referred [9]. Study of comprehensive survey on the latest developments in service-oriented network virtualization for supporting Cloud computing, particularly from a perspective of network and Cloud convergence is addressed [10]. Task scientific computing and corresponding analysis is carried out with empirical performance evaluation of four commercial cloud computing services and issues regarding cloud for scientific computing [11]. The Raspberry PI details and the Raspbian OS which was required for Raspberry PI has been taken from this website [12].

\section{PROPOSED SYSTEM}

The proposed work makes use of advanced cloud technology in combination with RFID technology for library management which will be more economical, more flexible, user friendly and efficient compared to conventional ones. The proposed work replaces the computers being used only to keep record of data and instead the system makes use of ARM to act as a virtual computer to send data on the internet cloud. The receiving users are having the flexibility to use the equipment of their choice with only condition of internet connectivity. Furthermore, instead of conventional internet, proposed work introduces new technology called CLOUD for the same task which is having inherent advantages over conventional online system such as cost and efficiency as mentioned above. The objectives of the proposed work are

1. To read data of books out of RFID tags on books and ID of corresponding person using RFID reader

2. To display information on the display and interface keypad for user interface

3. To process the data on ARM11 to send it on internet cloud

4.To manage data on cloud

5. To access data from cloud using any equipment having internet connectivity

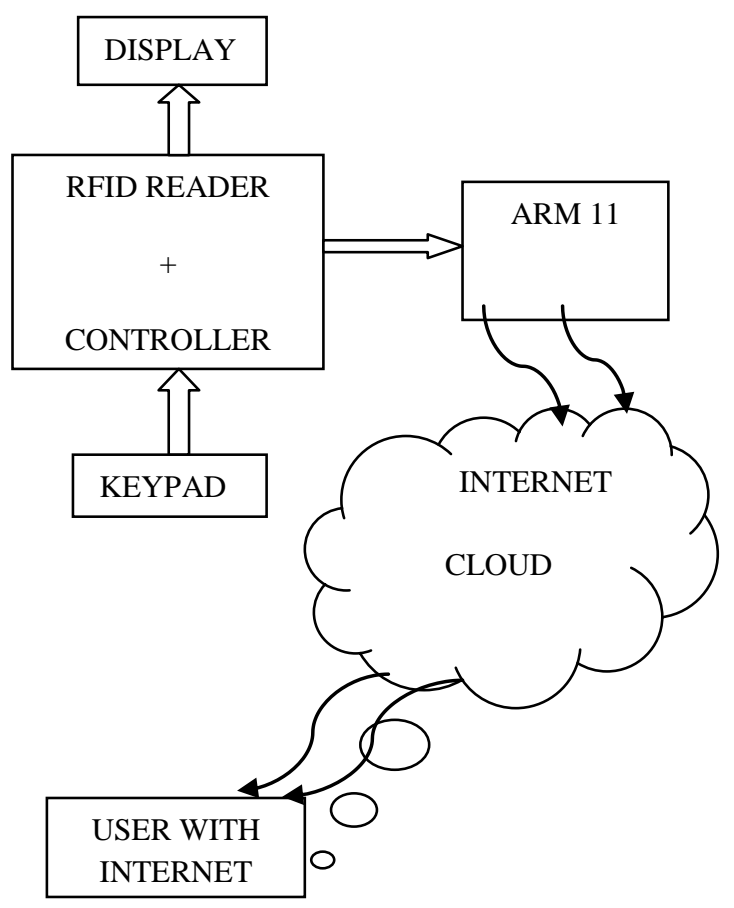

Fig 1: Proposed system block diagram

\section{RFID reader+ Controller:}

The system has been applied for library database access and management task for which RFID technology is implemented, where tags are appended to books and others are provided to users and data about books or users will be decoded using corresponding tags. RFID reader will read the data out of tags and will give it to the controller for processing, controller will have display and keypad connected to it and will be used for user interface.

2. ARM 11 controller:

The data sent by controller will now be processed in ARM11.The main advantages of ARM11 over other ARM 
versions are higher clock speeds, reduced heat production and lower overheating risk and the new instructions that are available. The ARM 11will be supported with SD card and Ethernet port for data storage and cloud connectivity respectively. It provides USB facility for internet dongle and external storage, if needed. The biggest advantage of using ARM 11 over conventional system is replacement of personal computers which were adding to excess costs and size. The ARM 11 is connected to cloud by means of socket programming which passes the data coming through the microcontroller circuitry. The ARM11 is used in the form of Raspberry PI model B[12] and hence accommodates all the above stated features in compact manner.

3. User with internet:

The authenticated user can access the details of data permitted to him remotely by using end equipment with internet connectivity. There will be two kinds of users accessing this data; first would be authenticated persons like librarian etc. and second would be the students or the users of library and hence two kinds of data would be there on cloud to be accessed. The proper IDs' and passwords would allow the appropriate user to access appropriate data.

\section{HARDWARE REQUIREMENT}

The 8051 controller is implemented to take the interrupt based output coming out of RFID reader, the protocol being Wiegand protocol. The RFID reader KR100E is implemented to satisfy the Wiegand protocol and being cost effective. The ARM 11 controller is implemented in the form of Raspberry PI kit. So in all the main Hardware used in this system is as follows..

1) KR 100E RFID reader

2) 8051 Microcontroller

3) Raspberry PI

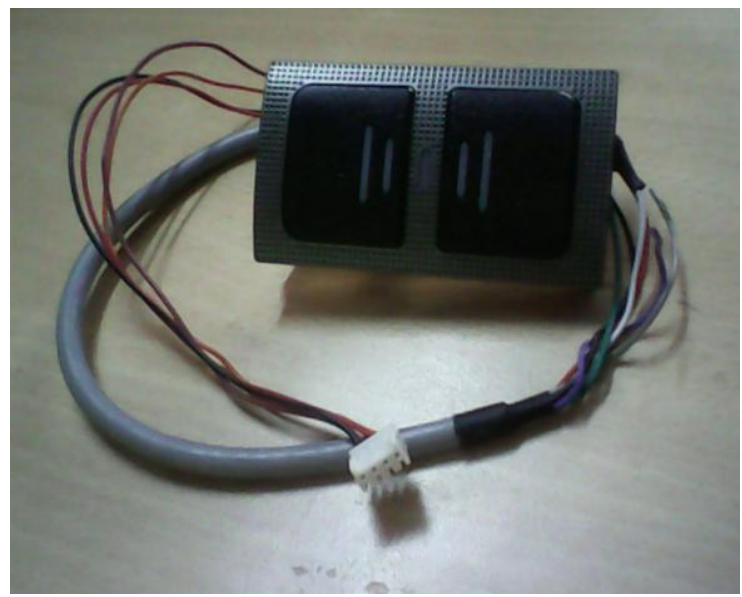

Fig 2: KR100 E RFID Reader

The (figure 2) shows the RFID reader used in the system namely KR100E supporting WIEGAND protocol. The (figure 3 ) shows the Raspberry PI model B used in the system to take the advantage of ARM11 and features like Ethernet port in compact manner. This Raspberry PI is used to replace the general purpose Desktop computers being used at libraries with a task specific computing unit

\section{RASPBERRY PI MODEL B}

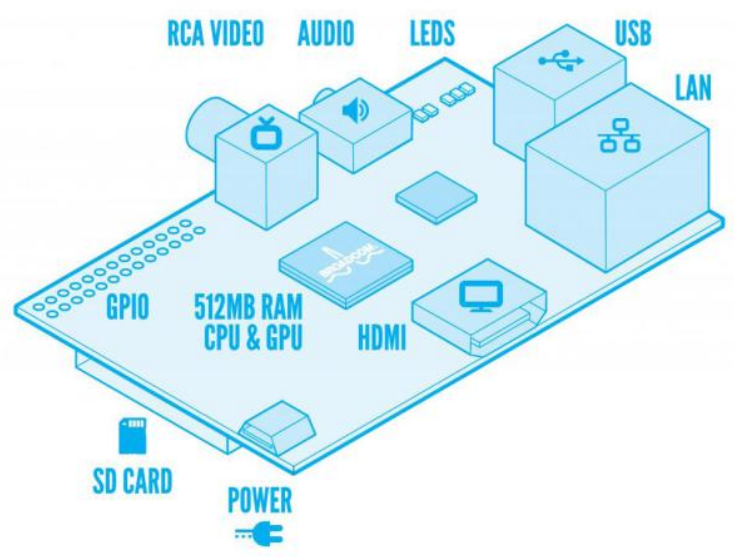

Fig 3: Raspberry PI model B [12]

\section{RESULTS}

From the resulting images, the corresponding RFID tags are shown (figure 4) where Book and User tags are shown separately placed. The controller display is shown in (figure 5 ) which shows the initial state of the system on power on. The controller circuitry is shown in (figure 6) where RFID reader is connected to controller by means of a four input connector and red light on reader indicates that it is waiting for tag to punch in. Further Raspberry Pi(ARM11) is shown in (figure 7) which is connected to controller circuit by means of serial connection to its GPIO. This Raspberry Pi is connected to cloud through Ethernet connection available. The authentication window is shown in (figure 8) which is on Cloud and allows users with proper usernames and passwords to view the data on cloud on go. The timings are shown in the table where the punching interval or the time taken by RFID reader for punch the tag is around $500 \mathrm{msec}$. The speed of data transfer from reader to controller unit is around 200 msec. Also the proximity in which the RFID reader can sense the RFID tag is $5 \mathrm{~cm}$ for KR $100 \mathrm{E}$.

Table 1. KR100 E Results

\begin{tabular}{|c|c|}
\hline Attributes & Values \\
\hline Punching interval & $0.5 \mathrm{sec}$ \\
\hline Speed & $0.2 \mathrm{sec}$ \\
\hline Proximity range & $5 \mathrm{~cm}$ \\
\hline Cloud data refresh rate & At every tag punch \\
\hline
\end{tabular}




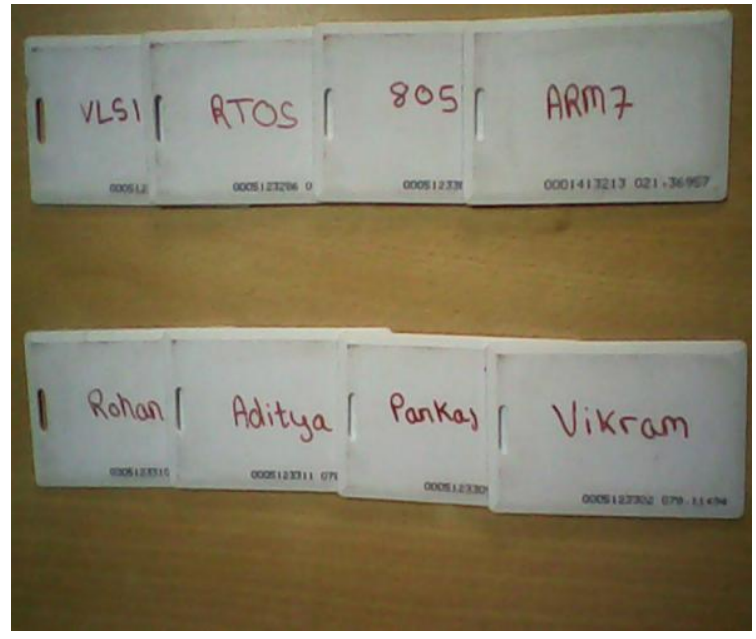

Fig 4: Book and User cards

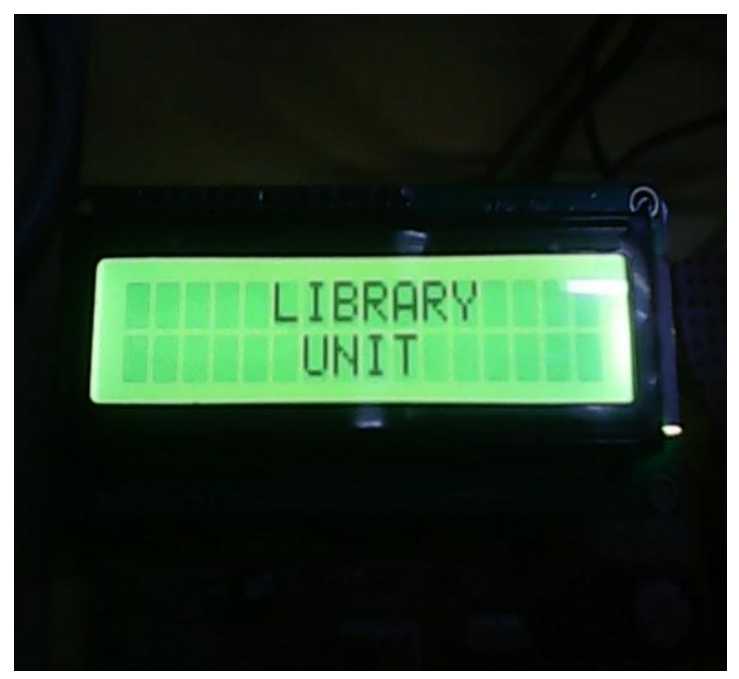

Fig 5: Controller display

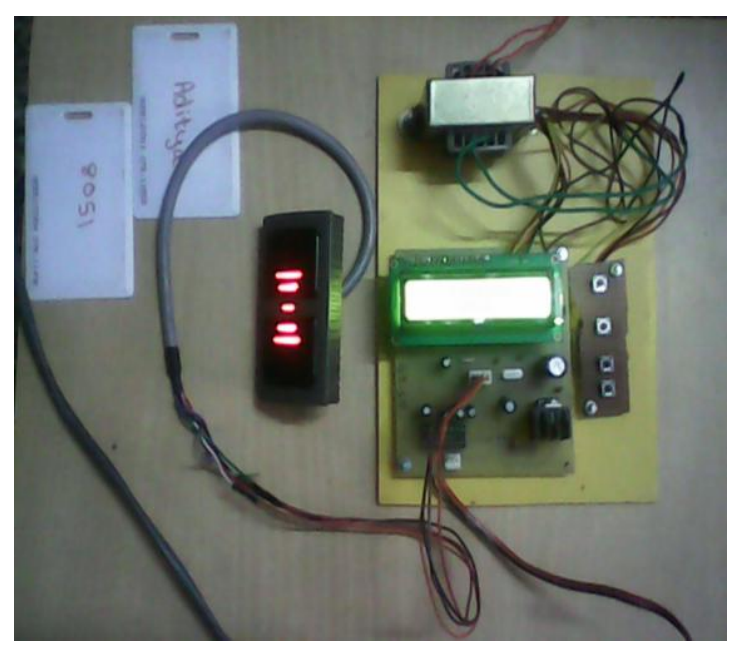

Fig 6: Controller Circuit

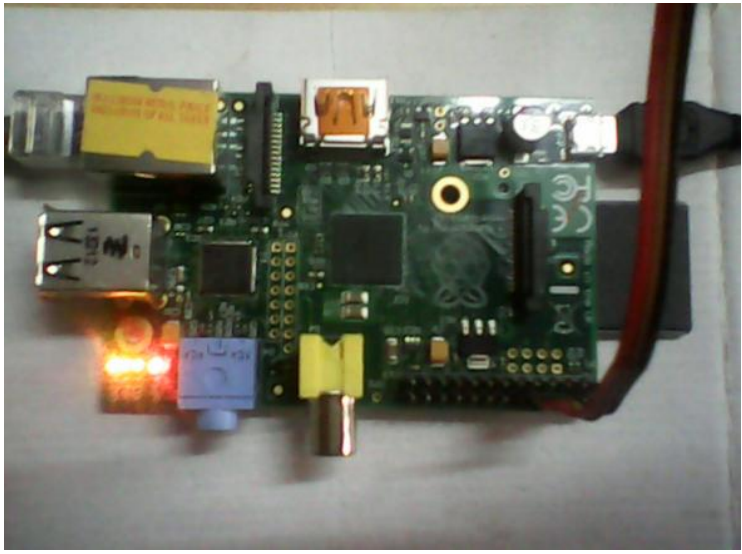

Fig 7: ARM11(Raspberry Pi)

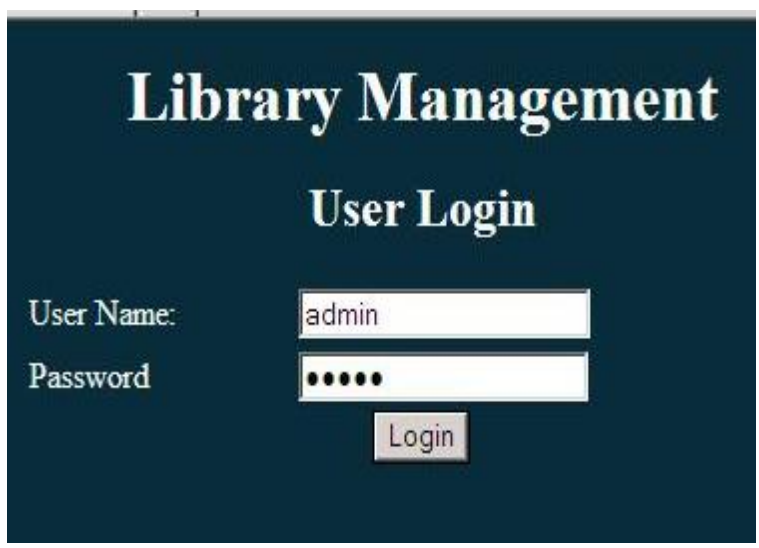

Fig 8: Authentication

\section{CONCLUSION}

Cloud is an area of research recently which must be employed in fields other than IT so as to utilize and exploit the inherent advantages of cloud to perform various tasks efficiently than by the conventional system being used today. The hardware and bulky equipments being used unnecessarily in various places could be efficiently replaced with the modern technologies. The new designs like ARM11 are likely to replace the desktop PC's in near future as what is being done in above system and so as space being used by such bulky equipments making them more and more task specific. The protocols like Wiegand protocol make the job of user easier by providing a flexible and reliable way of communication between readers and their corresponding controllers. The proposed system has made use of the latest technology available taking into account the cost optimization too to meet the application requirements and hence avoided higher precisions where not required. The proposed work can further be enhanced by making use of more compact RFID tags or even by replacing RFID technology for identification by the latest technology available then balancing the tradeoffs between cost and required precision. The system can also be optimized in future by replacing Raspberry PI with any circuit which will be more efficient then compared to Raspberry PI.

\section{ACKNOWLEDGMENTS}

I would like to express my sincere thanks to my project guide Dr. U.L.Bombale, Department of Technology, for his systematic approach in getting me familiarized with all the details needed for the project and constantly guiding me at every step. I am thankful to my family for their constant 
support and inspiration. At the end I would like to thank all persons who directly or indirectly helped me to complete this work.

\section{REFERENCES}

[1] Atta ur Rehman Khan, Mazliza Othman, Sajjad Ahmad Madani, IEEE Member, and Samee Ullah Khan, IEEE Senior Member: "A Survey of Mobile Cloud Computing Application Models", IEEE communications surveys IEEE 2013

[2] Vojislav B. Misic, Senior Member IEEE, Rajkumar Buyya, Dejan Milojicic,fellow IEEE, and Yong Cui: "Guest Editors' Introduction: Special Issue on Cloud Computing", IEEE Transaction on parallel and distributed systems, VOL. 24, NO. 6, JUNE 2013

[3] Yanjun Zuo: "Survivable RFID Systems: Issues, Challenges, and Techniques", IEEE transactions on systems,man and cybernatics- Part C: Applications and Reviews VOL. 40, NO. 4, JULY 2010

[4] Daniel J. Abadi: "Data Management in the Cloud: Limitations and Opportunities", Bulletin of the IEEE Computer Society Technical Committee on Data Engineering 2009 IEEE

[5] Óscar Diez, Andres Silva : "Govcloud: Using Cloud Computing in Public Organizations", IEEE technology and society magazine, March 2013

[6] Rahul Bhattacharyya, Student Member IEEE, Christian Floerkemeier, Member IEEE, and Sanjay Sarma, Member IEEE : "Low-Cost, Ubiquitous RFID-TagAntenna-Based Sensing" Proceedings of IEEE Vol. 98, No. 9, September 2010
[7] Po Yang, Wenyan Wu, Mansour Moniri, and Claude C. Chibelushi: "Efficient Object Localization Using Sparsely Distributed RFID Tags" IEEE transactions on industrial electronics VOL. 60, NO. 12, DECEMBER 2013

[8] A.Pravin Renold, Joshi Rani.R: "An Internet Based RFID Library Management System” Proceedings of 2013 IEEE Conferenceon Information and Communication Technologies (ICT 2013)

[9] Zeqiang Chen, Nengcheng Chen, Chao Yang, and Liping Di, Senior Member, IEEE: "Cloud computing Enabled Web Processing Service for Earth Observation Data Processing", IEEE journal of selected topics in applied earth observations and remote sensing,VOL 5, NO 6, DECEMBER 2012

[10] Qiang Duan, Yuhong Yan, and Athanasios V. Vasilakos: "A Survey on Service-Oriented Network Virtualization Toward Convergence of Networking and Cloud Computing", IEEE Transaction on Network and service management VOL. 9, NO. 4, DECEMBER 2012

[11] Alexandru Iosup, Member, IEEE, Simon Ostermann, M. NezihYigitbasi, Member, IEEE, Radu Prodan, Member, IEEE, Thomas Fahringer, Member, IEEE, and Dick H.J. Epema, Member, IEEE: "Performance Analysis of Cloud Computing Services for Many-Tasks Scientific Computing" IEEE Transaction on parallel and distributed systems, VOL. 22, NO. 6, JUNE 2011

[12] Raspberry PI Model B Available at website: http://www.raspberrypi.org 